\title{
The meaning of clinical ultrasound: clinical ultrasound and guidelines
}

\author{
Prof. Dr. Dieter Nürnberg
}

President DEGUM 2008-2010; Secretary of EFSUMB-Education Committee, Secretary of WFUMB

Ruppiner Kliniken GmbH, Neuruppin, Germany

What does the use of ultrasound in Romania have in common with its use in Germany? Both countries are committed to clinical ultrasound; meaning, that every medical discipline uses ultrasound as a primary, follow up and after-care method of examination in their field. Ultrasound, applied in a clinical setting, does not replace other imaging techniques, but integrates the clinical information, such as laboratory data, symptoms and the progression of the disease. Ultrasound becomes more meaningful with these additional data; it critically shortens diagnostics and monitors and leads the therapeutic approach in special ways. Other imaging techniques can follow and be used in a way to preserve resources and keep patients safe.

To use ultrasound in a clinical setting also means to teach ultrasound as part of the clinical training for the different medical disciplines, starting with the training for general practitioner to internist/gastroenterologist, to experts in medical imaging, the radiologist. Surely, special applications are a separate issue. But this training cannot start early enough and the young generations of doctors should be already confronted during medical school. EFSUMB (The European Federation for Ultrasound in Medicine and Biology) is currently developing a concept for students namely The European Course Book for Students (edit. R. Badea et al), available for everybody online which is an excellent contribution to this training. Clinical ultrasound does not mean that the comparison and the relationship with other imaging techniques will be abandoned. Especially, the radiologist as the master

Received Accepted

Med Ultrason

2013, Vol. 15, No 3, 171-172

Corresponding author: Prof. Dr. Dieter Nürnberg Ruppiner Kliniken $\mathrm{GmbH}$ Fehrbelliner Str. 38, 16816 Neuruppin, Germany Tel. 03391-393210, Fax 03391-393202

d.nuernberg@ruppiner-kliniken.de www.degum.de of imaging techniques, is a necessary and wanted partner in the evaluation of the different imaging methods. Ultrasound diagnosis competes with the other imaging methods and has an important function in interdisciplinary conferences such as oncology and angiology. The quality of ultrasound diagnostics depends not only on the quality of the equipment, but critically on the education of the examiner. But the education has to be graded, meaning, that the general practitioner and the young college in the emergency room can and have to answer other questions different from the highly equipped specialist in an ultrasound laboratory. Graded courses and educational programs, as well as the definition of the different levels (basic and advanced) facilitate the grading and secure a qualitatively high standard of ultrasound.

If and how ultrasound, as a non-radiation emitting clinical imaging method, can be established, is documented in the clinical guidelines. It took a long time to establish clinical ultrasound reasonably in German guidelines, although it has been practiced for years. Ultrasound has to compare itself to other methods available and to the outcome for the patient. In a variety of areas, it was possible to establish ultrasound in a sensible and adequate manner, such as in patients with colon and stomach carcinomas, with pancreas carcinomas or with chronic inflammatory bowel diseases.

DEGUM (Deutsche Gesellschaft für Ultraschall in der Medizin) has enforced its participation in the drafting guidelines and was very successful in recent months. Ultrasound and especially contrast enhanced ultrasound is now established in the German guidelines for the treatment of HCC, which is available online (www.awmf.de), in comparison to the European and American guidelines.

In recent years, I have visited the Romanian ultrasound congresses and I am impressed by the commitment and the scientific impact of the Romanian sonographers, especially in the scientific field on a European standard. Names such 
as Sporea, Badea, Săftoiu are internationally known. The contribution to the guidelines of EFSUMB in the area of contrast enhanced ultrasound and elastography are impressive. The number of Rumanian abstracts received for the European ultrasound congress 2013 in Stuttgart (www.euroson2013.de) places Romania on third place (after D and I)!
This present journal represents a high quality of applied clinical sonography and committed research in this field.

Congratulations for the Romanian ultrasound standards in this journal, a great development in European ultrasound. 